\title{
Generalized angular momentum transfer to up-converted photons via four-wave mixing in atomic gases
}

\author{
L. A. Mendoza-López, J. G. Acosta-Montes, I. F. Ángeles-Aguillón ๑, D. Sierra-Costa, Y. M. Torres, \\ R. Jáuregui, ${ }^{*}$ and D. Sahagún-Sánchez $\mathbb{Q}^{\dagger}$ \\ Departamento de Física Cuántica y Fotónica, Instituto de Física, Circuito Exterior, Universidad Nacional Autónoma de México, \\ Ciudad de México, CP 04510, México
}

(Received 22 February 2021; accepted 3 June 2021; published 20 August 2021)

\begin{abstract}
Experimental results on the phase transfer from structured beams to parametric light, generated by inducing four-wave mixing in an atomic gas, are reported. Near-infrared, Mathieu, and Gaussian pump beams yield a blue coherent Mathieu electromagnetic field and an infrared beam. Two complementary techniques enable full characterization of the up-converted modes. The unambiguous and efficient phase heritage of these complex quasipropagation invariant modes to up-converted photons is demonstrated. The presented data enable a quantitative study of dynamical variables related to Mathieu beams. These include elliptic angular momentum, a generalization of the orbital angular momentum of light, which is also up-converted through the nonlinear process.
\end{abstract}

DOI: 10.1103/PhysRevResearch.3.033170

\section{INTRODUCTION}

Four-wave mixing (FWM) in atomic gases is one of the most promising nonlinear processes for generating light with tailored classical and quantum properties. Exquisite control over the output optical frequency as well as its polarization and orbital angular momentum (OAM) offers new perspectives for developments in applied and fundamental contemporary physics. In particular, FWM in the diamond configuration can generate light with frequencies that are difficult to produce with common lasers [1-4]. In addition, the transfer of OAM from the pump beams to the atoms [5], and its retrieval [6], has introduced a new perspective of structured light interacting with matter. Conversion of Laguerre-Gauss beams via FWM has shown that OAM can be a valuable dynamical variable for the implementation of quantum engineering protocols using atomic gases [7-9]; the topological charge $m$ of an optical vortex is transferable within the $|m| \leqslant 30$ range [9] and the experiments evidence that the two spontaneously emitted photons in a FWM process may be entangled in this variable [10]. These correlations involve high-dimensional Hilbert spaces useful for enhanced quantum information management [11-13], with applications in quantum memories and imaging $[14,15]$, atom trapping and guiding [16,17], atomic state preparation [18], interactionfree measurements [19], and quantum telecommunications protocols [20]. Less studied alternatives to OAM are the dynamical properties resulting from the symmetry of other

\footnotetext{
*rocio@fisica.unam.mx

†sahagun@fisica.unam.mx
}

Published by the American Physical Society under the terms of the Creative Commons Attribution 4.0 International license. Further distribution of this work must maintain attribution to the author(s) and the published article's title, journal citation, and DOI. structured optical fields [21-25]. An example is the ideally propagating invariant Mathieu beams with elliptic-cylindrical symmetry $[21,26]$, which carry elliptic angular momentum (EAM), which is determined by the ellipticity of the corresponding coordinate system, the parity of the beam, and its order [21-24]. Elliptic angular momentum is a generalization of OAM for finite ellipticity. The paraxial analog of Mathieu beams is Ince-Gauss (IG) beams. Laguerre-Gauss modes, which may carry well-defined OAM, correspond to the limit of zero-coordinate-system ellipticity; in the limit of infinite ellipticity IG beams become Hermite-Gauss modes [27]. Thus, the ellipticity is an additional parameter to control the classical and quantum correlations of electromagnetic fields that can be implemented using beams with elliptical symmetry. Currently, most theoretical analyses of such correlations are made in terms of OAM [28,29].

In this work we report experiments on EAM transfer from Mathieu pump beams to up-converted light via FWM in an atomic gas. We demonstrate the transfer of the complex phase structure from a near-infrared quasipropagation invariant Mathieu beam to a coherent blue beam. Analysis of the pump and the parametric light in their Fourier and configuration spaces enables a full quantitative characterization of the process. With these procedures it is possible to make a compact description of the up-converted modes by using geometric parameters of the adequate coordinate system. More importantly, our results show the transfer of the EAM-associated dynamical variable from the pump beams to the up-converted electromagnetic fields.

\section{MATHIEU BEAMS AND ELLIPTIC ANGULAR MOMENTUM}

We begin by revising the general properties of Mathieu beams. Mathieu elementary modes can be written in terms of scalar wave functions $\Psi(\vec{r}, t)$, which in turn result from the 
superposition of plane waves through their frequency $\alpha(\omega)$ and angular $\mathcal{S}\left(\theta_{\vec{k}}, \varphi_{\vec{k}}\right)$ spectra,

$$
\Psi(\vec{r}, t)=\int d \omega \alpha(\omega) \int d^{3} k \delta(\omega-c|\vec{k}|) e^{i \vec{k} \cdot \vec{r}-i \omega t} \mathcal{S}\left(\theta_{\vec{k}}, \varphi_{\vec{k}}\right) .
$$

The angular spectrum of Mathieu waves

$$
\mathcal{S}_{M}\left(\theta_{\vec{k}}, \varphi_{\vec{k}} ; a, q\right)=\mathcal{A}_{M ; a, q}\left(\varphi_{\vec{k}}\right) \delta\left(\sin \theta_{\vec{k}}-\kappa_{\perp} c / \omega\right) \frac{\left|\cos \theta_{\vec{k}}\right|}{\sin \theta_{\vec{k}}}
$$

is expressed by solutions of the Mathieu equation [21]

$$
\left(\frac{d^{2}}{d \varphi_{\vec{k}}^{2}}+a-2 q \cos 2 \varphi_{\vec{k}}\right) \mathcal{A}_{M ; a, q}\left(\varphi_{\vec{k}}\right)=0, \quad q=\left(\frac{h \kappa_{\perp}}{2}\right)^{2} .
$$

Elliptic coordinates, which simplify the description of Mathieu beams, exhibit two foci separated by a distance $2 h$ in any plane transverse to their main propagation direction. The accessible values of the constant $a$ [Eq. (3)] are discrete, even in free space, and define the order of the modes when set in increasing sequence. Mathieu elementary modes can be chosen to have a well-defined parity. They are then denoted by $c e\left(\varphi_{\vec{k}} ; a, q\right)$ and $\operatorname{se}\left(\varphi_{\vec{k}} ; a, q\right)$ for even and odd solutions, respectively. The $\delta$ factor in Eq. (2) guarantees cylindrical symmetry in the field by restricting the participating plane waves to those sharing a common modulus $\kappa_{\perp}$ of their transverse wave vector. Nevertheless, actual realizations of Mathieu beams involve a conic-shell volume within wave-vector space derived by replacing the $\delta$ distribution by a properly normalized Gaussian distribution [30],

$$
\delta\left(\sin \theta-\kappa_{\perp} c / \omega\right) \rightarrow \frac{1}{(c \sigma / \omega) \sqrt{2 \pi}} e^{-\left(\sin \theta-\kappa_{\perp} c / \omega\right)^{2} / 2(c \sigma / \omega)^{2}},
$$

with waist $\sigma$.

The transverse vectors $\vec{r}_{\perp}^{ \pm}$defining the foci in an elliptic coordinate system give rise to natural evaluation points for optical angular momentum along $z$. A group theory analysis shows that the natural dynamic variable, i.e., the EAM of an optical Mathieu field [21-24], is defined by an operator $\hat{\mathbb{L}}_{z}^{2}$ involving the geometric average of the standard angular momentum operator $\hat{L}_{z \pm}=\left[\left(\vec{r}-\vec{r}^{ \pm}\right) \times i \vec{\nabla}\right]_{z}$,

$$
\hat{\mathbb{L}}_{z}^{2}=\left(\frac{1}{2}\left[\hat{L}_{z+} \hat{L}_{z-}+\hat{L}_{z-} \hat{L}_{z+}\right]-\frac{h^{2}}{2} \nabla_{\perp}^{2}\right),
$$

where $\nabla_{\perp}^{2}=\partial_{x}^{2}+\partial_{y}^{2}$. The scalar Mathieu wave function $\Psi_{a q}(\vec{r}, t)$ satisfies

$$
\hat{\mathbb{L}}_{z}^{2} \Psi_{a q}(\vec{r}, t)=a \Psi_{a q}(\vec{r}, t)
$$

which gives a dynamical interpretation of the parameter $a$. The classical analog of $\hat{\mathbb{L}}_{z}^{2}$ is essential for understanding the mechanical evolution of confined particles in elliptic billiards [31] and that of microparticles immersed in elliptic optical tweezers [24,32]. This dynamic variable is also similar to an integral of motion for a single electron moving in the field of two fixed nuclei [33].

\section{FOUR-WAVE-MIXING UP-CONVERSION}

Standard discussions about the expected quantitative properties of photons generated from nonlinear processes are based on phase-matching conditions. Within a perturbative analysis and for the FWM process under consideration, the steady-state amplitude for spontaneous emission of microwaves with electric fields $E_{\mathrm{CML}}(\vec{r}) e^{-i \omega_{\mathrm{CML}} t}$ and blue photons with $E_{\mathrm{CBL}}(\vec{r}) e^{-i \omega_{\mathrm{CBL}} t}$ is proportional to the functional

$$
\begin{aligned}
\mathfrak{F}\left[\vec{E}_{\mathrm{CML}}, \vec{E}_{\mathrm{CBL}}\right] & \\
= & \int_{-\infty}^{\infty} d t e^{i\left(\omega_{\mathrm{CML}}+\omega_{\mathrm{CBL}}\right) t} \int d^{3} r \vec{d}_{\mathrm{CML}}^{*} \\
& \cdot \vec{E}_{\mathrm{CML}}^{*}(\vec{r}) \vec{d}_{\mathrm{CBL}}^{*} \cdot \vec{E}_{\mathrm{CBL}}^{*}(\vec{r}) \Omega_{p_{1}}(\vec{r}, t) \Omega_{p_{2}}(\vec{r}, t) f_{A}(\vec{r}, t),
\end{aligned}
$$

where $\vec{d}_{\mathrm{CML}}$ and $\vec{d}_{\mathrm{CBL}}$ are the dipole moments of the atomic transitions in the spontaneous decays, $f_{A}(\vec{r}, t)$ is the atomic density, and the Rabi frequencies $\Omega_{p_{1}, p_{2}}(\vec{r}, t)$ encode the space-time structure of the pump beams. For ideal monochromatic $p_{1}$ and $p_{2}$ beams and assuming $f_{A}(\vec{r}, t)=f_{A}(\vec{r})$, the temporal integral yields

$$
\omega_{p_{1}}+\omega_{p_{2}}=\omega_{\mathrm{CML}}+\omega_{\mathrm{CBL}}
$$

with frequency $\omega_{\mathrm{CML}}\left(\omega_{\mathrm{CBL}}\right)$ in the microwave (blue) region.

The classical [34] and quantum [35] descriptions of electromagnetic fields can be carried out using diverse structured modes as a basis. In quantum optics, they must be normalized according to Einstein's quantization rule. In general, the experimental scenario determines the most adequate basis set for a description of the relevant electromagnetic modes. For the case of FWM in a quasihomogeneous medium, a functional maximization of $\left|\mathfrak{F}\left[\vec{E}_{\mathrm{CML}}, \vec{E}_{\mathrm{CBL}}\right]\right|$ with respect to the $\vec{E}_{\mathrm{CML}}$ and $\vec{E}_{\mathrm{CBL}}$ fields would allow identification of the optimal basis for a compact description of the converted photons, given the spatial structure of the pump beams. For idealized plane waves $\Omega_{p_{1}, p_{2}}(\vec{r})=e^{i\left(\vec{k}_{1,2} \cdot \vec{r}-\omega_{1,2} t\right)}\left|\Omega_{p_{1}, p_{2}}\right|, \mathfrak{F}\left[\vec{E}_{\mathrm{CML}}, \vec{E}_{\mathrm{CBL}}\right]$ is maximized by converted plane waves with propagation vectors satisfying

$$
\vec{k}_{p_{1}}+\vec{k}_{p_{2}}=\vec{k}_{\mathrm{CML}}+\vec{k}_{\mathrm{CBL}}
$$

Another relevant example corresponds to idealized Bessel pump beams with topological charges $m_{1,2}$ and wave numbers $k_{z_{1}, z_{2}}$ along $z$. Then $\mathfrak{F}\left[\vec{E}_{\mathrm{CML}}, \vec{E}_{\mathrm{CBL}}\right]$ is maximized for converted Bessel photons fulfilling

$$
\begin{aligned}
& k_{z 1}+k_{z 2}=k_{z \mathrm{CML}}+k_{z \mathrm{CBL}}, \\
& m_{1}+m_{2}=m_{\mathrm{CML}}+m_{\mathrm{CBL}} .
\end{aligned}
$$

The phase-matching conditions can be interpreted as conservation laws valid in homogeneous and isotropic environments: Eq. (8) for energy, Eq. (9) for linear momentum, and Eq. (11) for OAM in the case of linearly polarized beams exhibiting a helical dislocation.

Note that both Bessel and plane waves constitute a complete basis set for the description of electromagnetic fields. Any of them can be used to describe a FWM process. The role of phase-matching conditions for complex electromagnetic fields written in terms of plane waves can be understood as follows. Each component of the electric field is expressed 
in terms of their angular spectra [Eq. (1)]. Normalizing the electromagnetic mode in the quantum realm gives rise to a factor that can be absorbed in the frequency spectrum coefficient $\alpha(\omega)$. The amplitude $\mathfrak{F}\left[\vec{E}_{\mathrm{CML}}, \vec{E}_{\mathrm{CBL}}\right]$ is then written as a continuous superposition of four photon processes where any single term has the structure

$$
\begin{aligned}
& \alpha_{p_{1}}\left(\omega_{1}\right) \mathcal{S}_{p_{1}}\left(\theta_{\vec{k}_{1}}, \varphi_{\vec{k}_{1}}\right) \alpha_{p_{2}}\left(\omega_{2}\right) \mathcal{S}_{p_{2}}\left(\theta_{\vec{k}_{2}}, \varphi_{\vec{k}_{2}}\right) \alpha_{\mathrm{CML}}^{*}\left(\omega_{3}\right) \\
& \quad \times \mathcal{S}_{\mathrm{CML}}^{*}\left(\theta_{\vec{k}_{3}}, \varphi_{\vec{k}_{3}}\right) \alpha_{\mathrm{CBL}}^{*}\left(\omega_{4}\right) \mathcal{S}_{\mathrm{CBL}}^{*}\left(\theta_{\vec{k}_{4}}, \varphi_{\vec{k}_{4}}\right) \\
& \quad \times e^{i\left(\vec{k}_{1}+\vec{k}_{2}-\vec{k}_{3}-\vec{k}_{4}\right) \cdot \vec{r}} e^{-i\left(\omega_{1}+\omega_{2}-\omega_{3}-\omega_{4}\right) t} .
\end{aligned}
$$

Integrated in time and space, each product gives rise to phasematching conditions similar to Eqs. (8) and (9). The modulus of Eq. (12) yields the probability of generating single photons with frequencies and wave vectors $\omega_{3,4}$ and $\vec{k}_{3,4}$. This probability is proportional to the product of the moduli of the angular spectra involved. It is this dependence on the angular spectra that supports the heritage of phase structure in the FWM process. Note that the individual probabilities could be measured by a selective detection of photons with the corresponding $\left(\omega_{i}, \vec{k}_{i}\right)$ properties.

An alternative scheme is to incorporate the boundary conditions established by a given experimental setup to select the most natural basis for describing the experimental outcomes. In this article we report on the heritage of even and odd Mathieu modes from pump to up-converted light through a FWM process in an atomic gas. The electronic structure of alkali-metal atoms allows the implementation of an effective four-level system that can be populated cyclically in a diamond configuration. Figure 1(a) depicts its realization in the experiments reported here: It is based on the ladder $5 S_{1 / 2} \rightarrow 5 P_{3 / 2} \rightarrow 5 D_{5 / 2}$ transition of ${ }^{87} \mathrm{Rb}$ excited by 780 $\mathrm{nm}\left(p_{1}\right)$ and 776-nm $\left(p_{2}\right)$ light. In this case, the frequencies generated by the cascade decay are separated by an order of magnitude [1]. The $5 D_{5 / 2}$ has a dominating decay path to the $6 P_{3 / 2}$ state giving rise to a $5-\mu \mathrm{m}$ electromagnetic field. This is followed by a $6 P_{3 / 2} \rightarrow 5 S_{1 / 2}$ decay that fluoresces at $420 \mathrm{~nm}$. The field radiated from the latter can be readily collimated and power optimized with an appropriate choice of the pump polarizations and frequency detunings $\delta_{1}$ and $\delta_{2}[3,36]$. In the experiments depicted here structured collimated blue light (CBL) was generated by a Gaussian $p_{1}$ beam; MathieuGaussian modes were encoded on $p_{2}$, taking into account previous results on phase structure heritage in wave-mixing experiments involving this diamond configuration [37].

Since in our experimental setup the pump beams are quasimonochromatic, satisfy the paraxial condition and are arranged collinearly, a reasonable ansatz is to take the converted modes as Ince-Gauss fields with a well-defined parity. Some consequences of doing so are direct. Up to Gouy-like phases, the electric field of the pump beams has a harmonic evolution $e^{i k_{z} z}$. Then Eqs. (8) and (10) should be approximately satisfied. Therefore, the up-and down-converted photons are emitted in a quasicollinear configuration. The transverse structure of the $p_{1}$ Gaussian beam is a superposition of plane waves with $\left|\vec{k}_{\perp}\right|$ centered at the zero value and the transverse structure of the $p_{2}$ Mathieu beams has a finite $\left|\vec{k}_{\perp}\right|$ center. Additionally, the up-converted photons have a wider range of available wave vectors than the down-converted photons. So

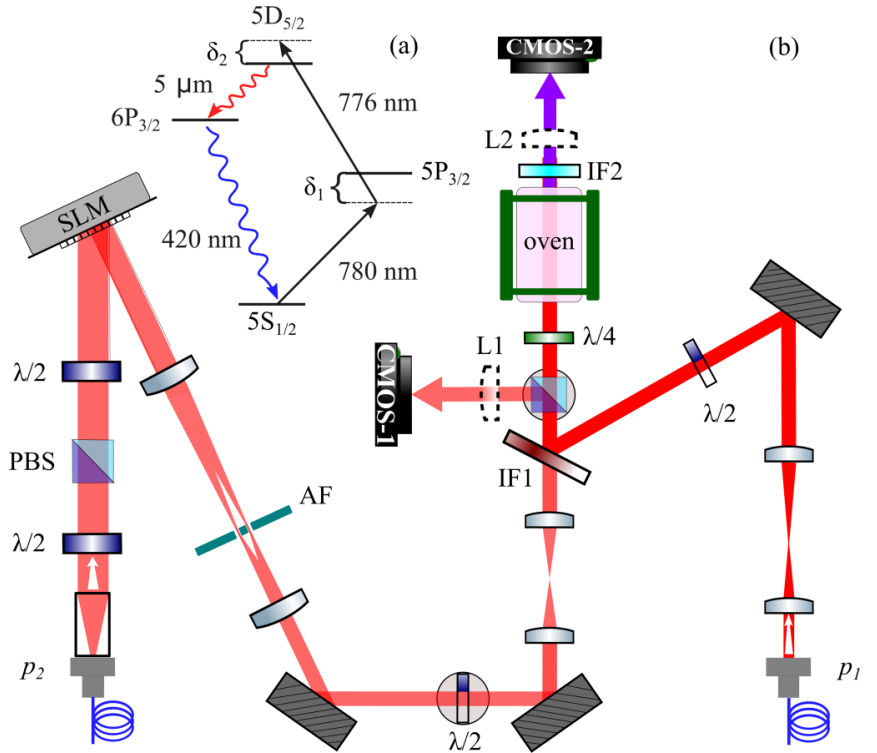

FIG. 1. (a) Implemented FWM diamond scheme. Two nearinfrared beams $p_{1}$ and $p_{2}$ excite a ladder transition that generates far infrared (CML) and blue (CBL) optical fields when atoms decay to their ground state. (b) Schematics of the experimental array. The pump light is prepared on the left and right sides. Both pump beams are overlapped for interaction with the atoms located inside an oven. Both $p_{2}$ and the CBL are analyzed with complementary metal-oxide semiconductor (CMOS) cameras 1 and 2. Labels denote optical elements as follows: $\lambda / 2$, half waveplate; PBS, polarizing beam splitter cube; SLM, spatial light modulator; AF, annular filter; IF, interference filter; $\lambda / 4$, quarter waveplate; L, Fourier lens; and CMOS, analysis camera.

the down-converted modes are expected to have a Gaussianlike structure with a soft transverse configuration, while the up-converted photons are compactly described within a Mathieu-Gauss (MG) basis.

\section{EXPERIMENT}

Our experimental apparatus is illustrated in Fig. 1(b). The two pump beams are delivered by optical fibers from their respective saturated spectroscopy setups (not shown). On the right, the $p_{1}$ Gaussian profile is expanded to a $4 \mathrm{~mm}$ diameter. On the left, $p_{2}$ is expanded to a $24 \mathrm{~mm}$ diameter for approximating a plane wave. At this stage, both pump beams are linearly polarized. The desired Mathieu mode is imprinted on $p_{2}$ using a spatial light modulator (SLM) following the method proposed in Ref. [38]. Notice that for even and odd Mathieu beams, the corresponding transverse phase is either $\phi_{\Psi}=0$ or $\phi_{\Psi}=\pi$ since the Mathieu functions are real. The first telescope on the $p_{2}$ path was used to match the $p_{1}$ diameter and to spatially filter its remaining Gaussian structure with the annular filter. The second telescope has unitary amplification and extends the quasi-invariant propagation of the generated MG beam. Both $p_{1}$ and $p_{2}$ beams are combined at interference filter IF1 and sent through a 75-mm-long spectroscopy cell with a natural rubidium mix.

In these experiments we kept $p_{1}$ locked to the $5 S_{1 / 2}, F=$ $2 \rightarrow 5 P_{3 / 2}, F=3$ transition $\left(\delta_{1}=0\right)$; although local maxima 

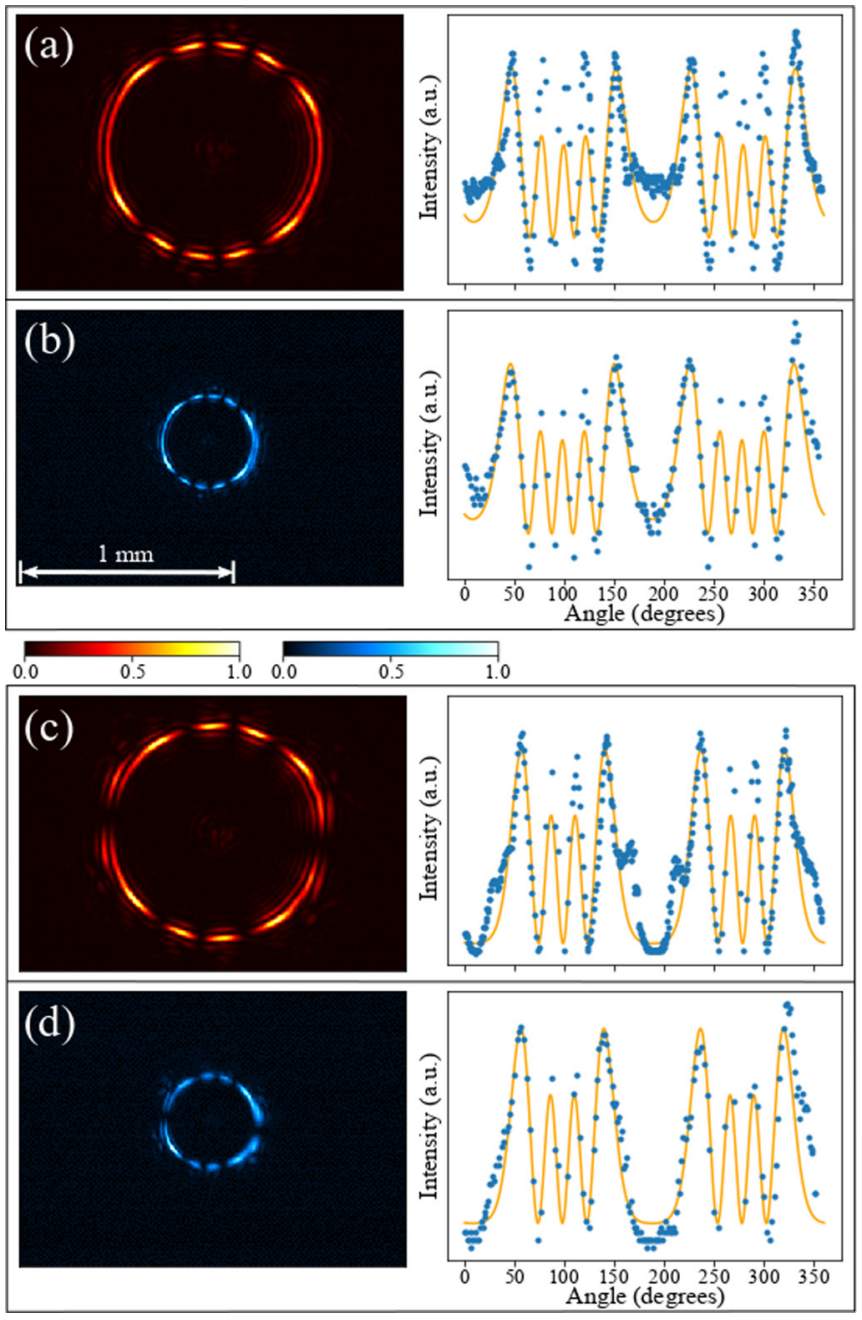

FIG. 2. Illustrative experimental Fourier rings and their corresponding measured (dots) and theoretical (continuum line) angular spectra of (a) and (b) even and (c) and (d) odd Mathieu-Gauss beams with order $n=4$. Images in red were taken from the Fourier plane of $p_{2}$ MG modes. The transverse wave vector $\kappa_{\perp}$ is measured using the data obtained from the images in the left column, while the $q$ parameter of the Mathieu modes is inferred from the data corresponding to the right column images.

of the CBL power were found at $\delta_{2}= \pm 6 \mathrm{MHz}$, most of the experiments were done with $p_{2}$ resonant to the $5 P_{3 / 2}, F=$ $3 \rightarrow 5 D_{5 / 2}, F=4$ transition. Further CBL power optimization is possible with larger values of both $\delta_{1}$ and $\delta_{2}$ [36], albeit coherence may be lost [39]. Optimization of the CBL power demands minor adjustments on the bichromatic beam alignment that are systematically different according to the mode parity. Two precautions enabled us to obtain reliable data even with relatively low pump power $(7 \mathrm{~mW}$ for both beams): making sure that both dipole transitions were cyclic by setting the same circular polarization on $p_{1}$ and $p_{2}$ [3] and achieving their maximum overlap by applying the Boyd criterion [40]. The data presented below were obtained at an atomic temperature of $80^{\circ} \mathrm{C}$. Based on absorption experiments in the $D 1$ line of ${ }^{87} \mathrm{Rb}$, we estimate this is equivalent to an atom density of approximately $7.4 \times 10^{10} \mathrm{~cm}^{-3}$.

\section{MEASUREMENTS IN WAVE-VECTOR SPACE}

As shown in Fig. 1(b), a polarizing beam splitter is located right before the oven to enable sampling of the $p_{2}$ intensity structure by blocking $p_{1}$. Its Fourier plane is readily captured by locating a lens L1 at its focal length from CMOS-1. Similarly, the undesired light for imaging the up-converted light is removed by interference filter IF2 and its Fourier space is imaged with CMOS-2 assisted by lens L2. Figure 2 shows the data obtained by this technique. Figures 2(a) and 2(c) show the Fourier rings corresponding to two illustrative examples of $p_{2}$ beams imprinted with even and odd MG modes, respectively. Both cases have order $n=4$ and an ideal $\kappa_{\perp}=32.4 \mathrm{~mm}^{-1}$ imprinted by the SLM. Figures 2(b) and 2(d) show the Fourier rings of the corresponding up-converted light. The standard procedure to measure $\kappa_{\perp}$ is to divide the ring average radius by $f \lambda$, where $f$ is the focal length of the Fourier lens and $\lambda$ is the wavelength of the beam [41]. The width of the rings $\sigma$ [Eq. (4)] is also measured. We write $\kappa_{\perp} \pm \sigma$ to report the results. For the even mode shown in Figs. 2(a) and 2(b), $\kappa_{\perp}^{p_{2}}=32.4 \pm 0.3 \mathrm{~mm}^{-1}$ and $\kappa_{\perp}^{\mathrm{CBL}}=32.2 \pm 0.8 \mathrm{~mm}^{-1}$. Similar numbers were measured from the odd mode depicted in Figs. 2(c) and 2(d). We found consistency in the results for several input modes and external conditions such as temperature and detuning of the $p_{2}$ beam. These data confirm our hypothesis that the up-converted, rather than down-converted, beam inherits most of the transverse momentum $k_{\perp}$.

The Fourier space can be used to measure the $q$ parameter of the Mathieu modes, which can be extracted from the angular dependence of the data in Fig. 2. As an example, we show in Fig. 2(a) the normalized angular dependence for the even-mode rings on $p_{2}$ and in Fig. 2(b) that of the upconverted blue light. To measure $q$, a fit looking for an optimal angular Mathieu function is performed. An even Mathieu angular function of order 4 is the natural ansatz for these cases. Up to a proportionality constant related to the square
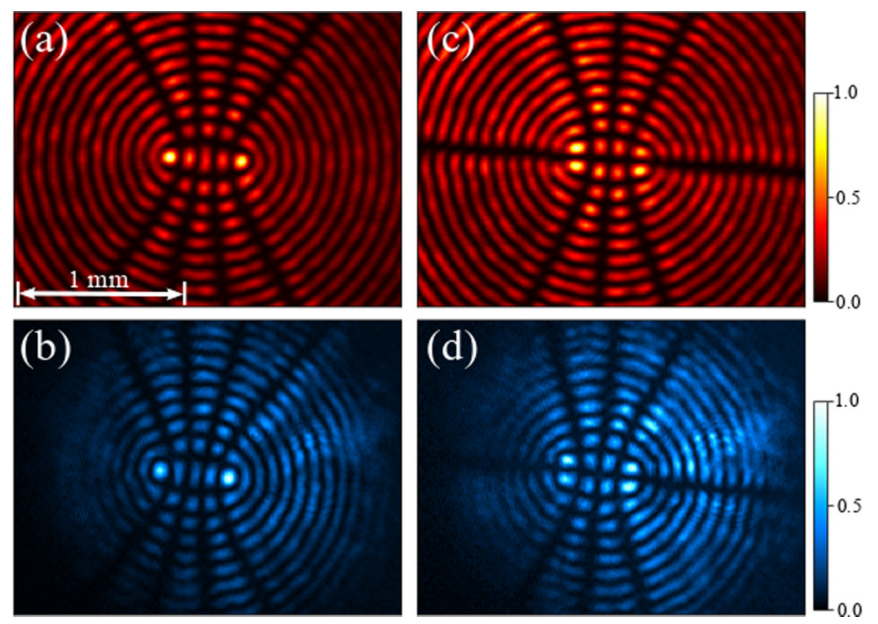

FIG. 3. Configuration space images of the beams depicted in Fig. 2. All beams shown occupy MG modes of order $n=4$ : (a) and (c) intensity profile of experimental $p_{2}$ even and odd beams and (b) and (d) respective up-converted profiles. From these images we extracted the interfocal distance $2 h$, which gives rise a second method to measure the parameter $q$ of the Mathieu modes. The intensity is normalized to its maximum value in each case. 
TABLE I. Measured half interfocal distance $h$, transverse wave vector $\kappa_{\perp}$, and geometrical factor $q$ for the Mathieu pump $p_{2}$ beam and the up-converted light depicted in Figs. 2 and 3. The $q$ has been evaluated using the $h$ measured from the configuration space images, while $q^{\prime}$ has been obtained from the Fourier space images. From them, the characteristic constants $a$ and $a^{\prime}$ are numerically estimated. The notation for a variable $x \pm \Delta x$ takes into account not just the systematic errors, but also the information arising from the finite width of the rings in wave-vector space whenever it is necessary.

\begin{tabular}{|c|c|c|c|c|}
\hline \multirow[b]{2}{*}{ Parameter } & \multicolumn{2}{|c|}{ Even Mathieu mode } & \multicolumn{2}{|c|}{ Odd Mathieu mode } \\
\hline & $p_{2}$ & $\mathrm{CBL}$ & $p_{2}$ & CBL \\
\hline$h(\mathrm{~mm})$ & $0.28 \pm 0.01$ & $0.29 \pm 0.01$ & $0.28 \pm 0.01$ & $0.28 \pm 0.01$ \\
\hline$\kappa_{\perp}\left(\mathrm{mm}^{-1}\right)$ & $32.4 \pm 0.3$ & $32.2 \pm 0.8$ & $32.4 \pm 0.3$ & $32.6 \pm 0.8$ \\
\hline$q$ & $20.7 \pm 2.2$ & $22.0 \pm 3.0$ & $19.9 \pm 2.3$ & $20.2 \pm 2.9$ \\
\hline$q^{\prime}$ & $20.6 \pm 1.7$ & $21.4 \pm 1.0$ & $20.8 \pm 1.5$ & $20.3 \pm 1.0$ \\
\hline$a$ & $27.5 \pm 0.4$ & $27.6 \pm 0.2$ & $15.7 \pm 0.7$ & $15.2 \pm 1.2$ \\
\hline$a^{\prime}$ & $27.6 \pm 0.3$ & $27.8 \pm 0.1$ & $15.0 \pm 0.7$ & $15.4 \pm 0.4$ \\
\hline
\end{tabular}

root of the intensity and an angular shift intrinsic to the beam orientation, the resulting $q$ parameters are $q_{p_{2}}=20.6 \pm 1.7$ and $q_{\mathrm{CBL}}=21.4 \pm 1.0$, so the characteristic values that determine $\hat{\mathbb{L}}_{z}^{2}$ are $a_{p_{2}}=27.6 \pm 0.3$ and $a_{\mathrm{CBL}}=27.8 \pm 0.1$ for the $p_{2}$ and the CBL mode, respectively. Thus, with this analysis, it is confirmed that the up-converted Mathieu mode inherits from $p_{2}$ both its geometric parameter $q$ and its elliptic angular momentum $\hat{\mathbb{L}}_{z}^{2}$.

\section{MEASUREMENTS IN CONFIGURATION SPACE}

In the configuration space we find a promising alternative for measuring geometrical properties such as $2 h$, a parameter directly involved in the definition of $\hat{\mathbb{L}}_{z}^{2}$ [Eq. (5)]. Figures 3(a) and 3(c) show images taken by CMOS-1 without L2; Figs. 3(b) and 3(d) show the up-converted beam profile without the Fourier lens. The images were also taken for even and odd Mathieu modes of order 4. To measure $2 h$, we first allocated the semimajor axis of the elliptic mode and then identified the intensity maxima and minima. These parameters were fit to ideal beam expectations. For the beam depicted in Fig. 3(a) we found $h_{p_{2}}=0.28 \pm 0.01 \mathrm{~mm}$. By inserting this value, together with the $\kappa_{\perp ; p_{2}}$ previously measured, into Eq. (3) we can compare both methods for measuring the parameter $q$, which yields $20.7 \pm 2.2$ in this case. We performed the same measurement over the FWM transferred structure yielding $h_{\mathrm{CBL}}=0.29 \pm 0.01 \mathrm{~mm}$ and $q_{\mathrm{CBL}}=22.0 \pm 3.0 \mathrm{~mm}$. For comparison, Table I gathers all the mode parameters measured with the two methods.

\section{CONCLUSION}

We have demonstrated that complex quasipropagation invariant modes can be up-converted through a FWM process in atomic gases. For this, we introduced theoretical and experimental methods giving quantitative insight into the pump and the generated light. Intensity and phase structure analyses, in both Fourier and configuration spaces, are established methods in nonlinear optics using crystals. As we have shown, they offer a promising alternative for research involving atomic gases as well. We investigated on the structure transfer from infrared $(776 \mathrm{~nm})$ to blue $(420 \mathrm{~nm})$ light. This analysis allowed us to measure parameters giving a compact description of the pump and up-converted light in terms of Mathieu-Gauss modes. Both the transverse wave vector modulus $\kappa_{\perp}$ and the geometric parameter $q$ were confirmed to have the same values within error bars for the $p_{2}$ and the parametric blue light. The characteristic parameter $a$, which determines the order of even and odd Mathieu modes, and the value for the optical elliptic angular momentum $\hat{\mathbb{L}}_{z}^{2}$ were also inferred. Our data give conclusive evidence that $\hat{\mathbb{L}}_{z}^{2}$ is inherited together with the other light characteristics. We have demonstrated transfer of EAM; we have shown that dynamical variables other than linear momentum and orbital angular momentum can be transferred to photons using nonlinear optics phenomena in atomic gases. Equivalent transfer is expected for other structured light fields with different symmetries. Therefore, this work provides a foundation for exciting research activity on the design of light sources with classical and quantum coherence, frequency bandwidth, and polarization control using atomic gases.

\section{ACKNOWLEDGMENTS}

We thank the Consejo Nacional de Ciencia y Tecnología (CONACyT) for support through the National Laboratories Program, Grants No. 280181, No. 293471, and No. 299057 , and through the Basic Science Grant SEP-CONACyT No. 285387. L.A.M.-L., J.G.A.-M., and I.F.Á.-A. thank CONACyT for their postgraduate study fellowships. Y.M.T. thanks DGAPA-UNAM for postdoctoral support. We also thank DGAPA-UNAM for support through PAPIIT Grants No. IN108018, No. IN-103020, and No. IN106821 and PIIF-UNAM. R.J. and D.S.-S. thank Robert Sewell for carefully reviewing the manuscript. R.J. thanks Ricardo Guitérrez-Jáuregui for discussions regarding this project.
[1] A. S. Zibrov, M. D. Lukin, L. Hollberg, and M. O. Scully, Efficient frequency up-conversion in resonant coherent media, Phys. Rev. A 65, 051801(R) (2002).
[2] T. Meijer, J. D. White, B. Smeets, M. Jeppesen, and R. E. Scholten, Blue five-level frequency-upconversion system in rubidium, Opt. Lett. 31, 1002 (2006). 
[3] A. M. Akulshin, A. I. Sidorov, P. Hannaford, and R. J. McLean, Coherent and collimated blue light generated by four-wave mixing in $\mathrm{Rb}$ vapour, Opt. Express 17, 22861 (2009).

[4] M. Lam, S. B. Pal, T. Vogt, C. Gross, M. Kiffner, and W. Li, Collimated UV light generation by two-photon excitation to a Rydberg state in Rb vapor, Opt. Lett. 44, 2931 (2019).

[5] J. W. R. Tabosa and D. V. Petrov, Optical Pumping of Orbital Angular Momentum of Light in Cold Cesium Atoms, Phys. Rev. Lett. 83, 4967 (1999).

[6] S. Barreiro and J. W. R. Tabosa, Generation of Light Carrying Orbital Angular Momentum via Induced Coherence Grating in Cold Atoms, Phys. Rev. Lett. 90, 133001 (2003).

[7] G. Walker, A. S. Arnold, and S. Franke-Arnold, Trans-Spectral Orbital Angular Momentum Transfer via Four-Wave Mixing in Rb Vapor, Phys. Rev. Lett. 108, 243601 (2012).

[8] A. M. Akulshin, E. E. Mikhailov, I. Novikova, R. J. McLean, and S. A. Suslov, Arithmetic with optical topological charges in stepwise-excited Rb vapor, Opt. Lett. 41, 1146 (2016).

[9] A. Chopinaud, M. Jacquey, B. Viaris de Lesegno, and L. Pruvost, High helicity vortex conversion in a rubidium vapor, Phys. Rev. A 97, 063806 (2018).

[10] R. F. Offer, D. Stulga, E. Riis, S. Franke-Arnold, and A. S. Arnold, Spiral bandwidth of four-wave mixing in $\mathrm{Rb}$ vapour, Nat. Commun. Physics 1, 84 (2018).

[11] S. Gröblacher, T. Jennewein, A. Vaziri, G. Weihs, and A. Zeilinger, Experimental quantum cryptography with qutrits, New J. Phys. 8, 75 (2006).

[12] N. K. Langford, R. B. Dalton, M. D. Harvey, J. L. O’Brien, G. J. Pryde, A. Gilchrist, S. D. Bartlett, and A. G. White, Measuring Entangled Qutrits and their Use for Quantum Bit Commitment, Phys. Rev. Lett. 93, 053601 (2004).

[13] D. Gutiérrez-López, M. Maldonado-Terrón, R. J. Hernández, V. Vicuña-Hernández, R. Ramírez-Alarcón, H. Cruz-Ramírez, R. Jáuregui, and A. B. U'Ren, Spatial control of spontaneous parametric down-conversion photon pairs through the use of apertured Bessel-Gauss pump beams, Phys. Rev. A 100, 013802 (2019).

[14] M. Shuker, O. Firstenberg, R. Pugatch, A. Ron, and N. Davidson, Storing Images in Warm Atomic Vapor, Phys. Rev. Lett. 100, 223601 (2008).

[15] D.-S. Ding, Z.-Y. Zhou, B.-S. Shi, and G.-C. Guo, Singlephoton-level quantum image memory based on cold atomic ensembles, Nat. Commun. 4, 2527 (2013).

[16] K. Volke-Sepúlveda and R. Jáuregui, All-optical 3D atomic loops generated with Bessel light fields, J. Phys. B 42, 085303 (2009).

[17] B. M. Rodríguez-Lara and R. Jáuregui, Single structured light beam as an atomic cloud splitter, Phys. Rev. A 80, 011813(R) (2009).

[18] R. Jáuregui, Control of atomic transition rates via laser-light shaping, Phys. Rev. A 91, 043842 (2015).

[19] J.-P. Wolf and Y. Silberberg, Quantum optics: Spooky spectroscopy, Nat. Photon. 10, 77 (2016).

[20] T. Chanelière, D. N. Matsukevich, S. D. Jenkins, T. A. B. Kennedy, M. S. Chapman, and A. Kuzmich, Quantum Telecommunication Based on Atomic Cascade Transitions, Phys. Rev. Lett. 96, 093604 (2006).
[21] C. P. Boyer, E. G. Kalnins, and W. Miller, Symmetry and separation of variables for the Helmholtz and Laplace equations, Nagoya Math. J. 60, 35 (1976).

[22] M. A. Bandres and J. C. Gutiérrez-Vega, Elliptical beams, Opt. Express 16, 21087 (2008).

[23] K. Volke-Sepúlveda and E. Ley-Koo, General construction and connections of vector propagation invariant optical fields: TE and TM modes and polarization states, J. Opt. A 8, 867 (2006).

[24] B. M. Rodríguez-Lara and R. Jáuregui, Dynamical constants for electromagnetic fields with elliptic-cylindrical symmetry, Phys. Rev. A 78, 033813 (2008).

[25] C. L. Hernandez-Cedillo, S. Bernon, H. Hattermann, J. Fortágh, and R. Jáuregui, Scattering of dilute thermal atom clouds on optical Weber beams, Phys. Rev. A 87, 023404 (2013).

[26] J. C. Gutiérrez-Vega, M. D. Iturbe-Castillo, and S. ChávezCerda, Alternative formulation for invariant optical fields: Mathieu beams, Opt. Lett. 25, 1493 (2000).

[27] J. C. Gutiérrez-Vega and M. A. Bandres, Ince-Gaussian beams, Opt. Lett. 29, 144 (2004).

[28] W. N. Plick, M. Krenn, R. Fickler, S. Ramelow, and A Zeilinger, Quantum orbital angular momentum of elliptically symmetric light, Phys. Rev. A 87, 033806 (2013).

[29] B. Baghdasaryan and S. Fritzsche, Enhanced entanglement from Ince-Gaussian pump beams in spontaneous parametric down-conversion, Phys. Rev. A 102, 052412 (2020).

[30] A. Dartora, M. Zamboni-Racheda, K. Nóbregaa, E. Recamib, and H. Hernández-Figueroa, General formulation for the analysis of scalar diffraction-free beams using angular modulation: Mathieu and Bessel beams, Opt. Commun. 222, 75 (2003).

[31] A. J. S. Traiber, A. J. Fendrik, and M. Bernath, The rotating elliptic billiard and a signature of quantum chaos, J. Phys. A: Math. Gen. 23, L305 (1990).

[32] C. López-Mariscal, J. C. Gutiérrez-Vega, G. Milne, and K. Dholakia, Orbital angular momentum transfer in helical Mathieu beams, Opt. Express 14, 4182 (2006).

[33] H. A. Erikson and E. L. Hill, A note on the one-electron states of diatomic molecules, Phys. Rev. 75, 29 (1949).

[34] H. Rubinsztein-Dunlop, A. Forbes, M. V. Berry, M. R. Dennis, D. L. Andrews, M. Mansuripur, C. Denz, C. Alpmann, P. Banzer, T. Bauer, E. Karimi, L. Marrucci, M. Padgett, M. Ritsch-Marte, N. M. Litchinitser, N. P. Bigelow, C. Rosales-Guzmán, A. Belmonte, J. P. Torres, T. W. Neely et al., Roadmap on structured light, J. Opt. 19, 013001 (2016).

[35] R. Jáuregui and S. Hacyan, Quantum-mechanical properties of Bessel beams, Phys. Rev. A 71, 033411 (2005).

[36] A. Vernier, A. S. Arnold, E. Riis, and S. Franke-Arnold, Enhanced frequency up-conversion in Rb vapor, Opt. Express 18 , 17020 (2010).

[37] A. M. Akulshin, R. J. McLean, E. E. Mikhailov, and I. Novikova, Distinguishing nonlinear processes in atomic media via orbital angular momentum transfer, Opt. Lett. 40, 1109 (2015).

[38] R. J. Hernández-Hernández, R. A. Terborg, I. RicardezVargas, and K. Volke-Sepúlveda, Experimental generation of 
Mathieu-Gauss beams with a phase-only spatial light modulator, Appl. Opt. 49, 6903 (2010).

[39] A. M. Akulshin, D. Budker, and R. J. McLean, Parametric wave mixing enhanced by velocity-insensitive two-photon excitation in Rb vapor, J. Opt. Soc. Am. B 34, 1016 (2017).
[40] R. W. Boyd, M. S. Malcuit, D. J. Gauthier, and K. Rząewski, Competition between amplified spontaneous emission and the four-wave-mixing process, Phys. Rev. A 35, 1648 (1987).

[41] J. W. Goodman, Introduction to Fourier Optics, 3rd ed. (Roberts, Greenwood Village, 2005). 